УДК 616.65-006.6;61:575

СКоллектив авторов

$$
\begin{gathered}
\text { СТАТУС МЕТИЛИРОВАНИЯ ГЕНОВ GSTP1, АРС И RASSF1 В ОБРАЗЦАХ } \\
\text { РАКА ПРЕДСТАТЕЛЬНОЙ ЖЕЛЕЗЫ ЧЕЛОВЕКА: СРАВНИТЕЛЬНЫЙ АНАЛИЗ } \\
\text { ДИАГНОСТИЧЕСКОЙ ИНФОРМАТИНОСТИ МЕТОДОВ МS-НRМ И ГИБРИДИАЦИИ } \\
\text { НА ДНК-ЧИПАХ ILLUMINA INFINIUМ НUМАNМЕТНYLATION450 ВЕАDСНIP }
\end{gathered}
$$

\author{
Л.О. Скородумова ${ }^{1 *}$ К.А. Бабалян ${ }^{2}$ Р. Султанов ${ }^{2}$ А.О. Васильев ${ }^{3}$ А.В. Говоров ${ }^{3}$, Д.Ю. Пушкарь \\ Е.А. Прилепская ${ }^{3}$ С.А. Даниленко ${ }^{1}$ Э.В. Генерозов ${ }^{1}$, А.К. Ларин ${ }^{1}$, Е.С. Кострюкова ${ }^{1,2}$, Е.И. Нарова \\ ${ }^{1}$ Федеральный научно-клинический центр физико-химической медицины \\ Федерального медико-биологического агентства, 119435, Москва, ул. Малая Пироговская, д. 1a; \\ тел.: +7(499)2464501; факс: +7(499)2464409; эл. почта: 1.skorodumova@rcpcm.org \\ ${ }^{2}$ Московский физико-технический институт (государственный университет), г. Долгопрудный \\ ${ }^{3}$ Московский государственный медико-стоматологический университет имени А.И. Евдокимова, Москва
}

В настоящее время существует потребность в молекулярных маркерах рака предстательной железы (РПЖ), уточняющих стратификацию пациентов по категориям риска и дополняющих факторы клинического прогноза. Одним из молекулярных процессов, происходящих при развитии РПЖ, является изменение метилирования ДНК. Для оценки метилирования генов в лабораторной диагностике может быть использована чувствительная к метилированию ПЦР с анализом кривой плавления высокого разрешения (Methylation-Sensitive High Resolution Melting - MS-HRM). Этот метод особенно перспективен с точки зрения небольшого количества требуемой ДНК (10 нг). На сегодняшний день накоплено много данных по изучению уровня метилирования ДНК в образцах РПЖ, полученных с использованием технологии ДНК-чипов “Infinium HumanMethylation450 BeadChip" (HM450). Однако неизвестно, согласуются ли результаты MS-HRM с данными чип-гибридизации. Целью данной работы был сравнительный анализ диагностической информативности методов MS-HRM и чип-гибридизации НM450 при определении степени метилирования генов GSTP1, APC и RASSF1 в образцах, полученных от больных РПЖ. Показано, что метод MS-HRM позволяет дифференцировать образцы опухолевой ткани РПЖ от образцов гистологически неизмененной ткани предстательной железы по уровню метилирования каждого из генов. Анализ данных чип-гибридизации подтвердил результаты, полученные с помощью MS-HRM. Разности уровней метилирования генов GSTP1, APC и RASSF1 между опухолевой тканью и гистологически неизмененной тканью у каждого больного РПЖ, определённые MS-HRM или гибридизацией на чипах, согласовались друг с другом. Таким образом, оценка уровня метилирования генов GSTP1, APC и RASSF1 с помощью MS-HRM может стать основой для разработки лабораторного теста, уточняющего диагностику РПЖ.

Ключевые слова: рак предстательной железы, метилирование ДНК, HRM, GSTP1, APC, RASSF1

DOI 10.18097/PBMC20166206708

\section{ВВЕДЕНИЕ}

Рак предстательной железы (РПЖ) является вторым по распространенности онкологическим заболеванием у мужчин в мире [1]. В России в 2015 году зарегистрировано 35540 больных с диагнозом РПЖ [2]. Окончательный диагноз РПЖ устанавливается на основании результатов гистологического исследования [3]. Пациенты с подозрением на РПЖ проходят процедуру биопсии под ультразвуковым контролем; пациенты с отрицательным результатом биопсии подвергаются ей повторно. Это приводит либо к задержке постановки правильного диагноза у пациентов с РПЖ, либо к ненужным инвазивным процедурам у пациентов без РПЖ [4]. Помимо этого, существует потребность в прогностических маркерах для пациентов с высоким риском рецидивирования РПЖ. Таким образом, актуальной является задача поиска методов диагностики РПЖ, уточняющих стратификацию пациентов по категориям риска.

Изменение метилирования $\mathrm{CpG}$ островков ДНК является одним из молекулярных процессов, обеспечивающих развитие опухоли [5]. Наиболее яркими проявлениями этого процесса являются гиперметилирование промоторов опухолевых супрессоров и деметилирование промоторов онкогенов [6]. Этот процесс характерен и для РПЖ, причем, скорее всего, он происходит уже на самых ранних стадиях развития опухоли [7]. Известно, что метилирование ДНК изменяется не только в опухолевых клетках РПЖ, но и в гистологически нормальной перитуморальной ткани предстательной железы, что может способствовать уточнению диагноза при исследовании биопсийных образцов [8].

Одним из методов анализа метилирования ДНК на уровне полного генома является гибридизация на ДНК-чипах. Например, микроматричный чип "Infinium HumanMethylation450 BeadChip array" (HМ450) позволяет оценить метилирование 98,9\% всех охарактеризованных генов (по базе данных UCSC RefGenes). C помощью гибридизации на ДНК-чипах можно выявить и использовать в дальнейшем в качестве потенциальных маркеров РПЖ большое количество $\mathrm{CpG}$ сайтов, отличающихся по степени метилирования в опухолевой и гистологически 


\section{ДИФФЕРЕНЦИАЛЬНОЕ МЕТИЛИРОВАНИЕ GSTP1, APC И RASSF1 В РПЖ}

неизменённой тканях предстательной железы. На данный момент опубликован ряд исследований РПЖ, в которых был использован этот подход [9-13]. Однако чип-гибридизация не подходит для рутинной лабораторной диагностики в связи со стоимостью, трудоёмкостью, а также необходимостью большого количества исходной ДНК (около 500 нг). Более удобными для лабораторной диагностики являются методы качественной и количественной метил-специфической ПЦР. Среди них чувствительная к метилированию ПЦР с анализом кривой плавления высокого разрешения (Methylation-Sensitive High Resolution Melting - MS-HRM). Этот метод требует небольшого количества ДНК (10 нг), что в дальнейшем может позволить перейти на малоинвазивные методы диагностики РПЖ [14]. Хотя гибридизация на ДНК-чипах является широко применяемым в научных исследованиях РПЖ методом со стандартизованным протоколом, неизвестно, насколько её результаты согласуются с данными MS-HRM.

Для изучения согласованности данных гибридизации на ДНК-чипах с данными MS-HRM были выбраны гены GSTP1, APC и RASSF1. Ранее метод MS-HRM не использовался для анализа уровня метилирования этих генов при РПЖ. Однако с помощью других методов метил-специфической ПЦР было показано, что статус метилирования промоторов генов GSTP1, APC и RASSF1 в биопсийных образцах ткани предстательной железы может иметь прогностическое значение $[4,15,16]$. Таким образом, целью данной работы был сравнительный анализ диагностической информативности методов MS-HRM и гибридизации на НM450 для определения степени метилирования генов GSTP1, APC и RASSF1 в образцах РПЖ.

\section{МЕТОДИКА}

\section{Клиническая группа}

Сбор образцов производился на базе кафедры урологии МГМСУ г. Москвы. В исследование было включено 8 пациентов (40-73 лет, средний возраст 62 года) после радикальной простатэктомии с ацинарной аденокарциномой, ранее не лечившихся по поводу РПЖ и давших информированное согласие на участие. Диагноз был верифицирован гистологически. У всех пациентов отмечалась периневральная инвазия. Данные пациенты включены в более крупное исследование, часть данных которого уже опубликована [17].

\section{Выделение ДНК из парафиновых срезов}

Для анализа метилирования использовали ДНК, полученную из послеоперационных образцов тканей предстательной железы. Послеоперационный материал был фиксирован в формалине и парафинирован, соответствующие тонкие срезы парафиновых блоков были охарактеризованы патоморфологом с выделением участка опухолевой и гистологически неизмененной прилегающей ткани. Из охарактеризованных участков срезов ткани предстательной железы каждого пациента было получено по одному образцу ДНК опухолевой ткани и ДНК гистологически неизмененной перитуморальной ткани - “нормы”. Депарафинизацию выполняли ксилолом, ДНК из парафинизированных тканей выделяли с помощью набора AllPrep DNA/RNA FFPE (“Qiagen”, Германия).

\section{Бисульфитная конверсия}

Bce образцы ДНК, проанализированные в данной работе, были подвергнуты процедуре бисульфитной конверсии. Под действием бисульфита натрия неметилированный цитозин дезаминируется в урацил. Урацил комплементарен аденину, поэтому в результате амплификации последовательности ДНК на месте неметилированного цитозина будет тимин. На 5-метилцитозин и 5-гидроксиметилцитозин бисульфит натрия не воздействует, поэтому метилированный цитозин в результате амплификации остаётся цитозином. Таким образом, становится возможным различить метилированные и неметилированные $\mathrm{CpG}$ сайты. Бисульфитная конверсия была выполнена с использованием набора EZ DNA Methylation kit ("ZymoResearch", СШA), согласно инструкции производителя.

\section{$M S-H R M$}

Праймеры для анализа статуса метилирования генов GSTP1, APC и RASSF1 с помощью MS-HRM были взяты из ранее опубликованных работ, в которых эти праймеры использовали в исследованиях опухолей молочной железы или колоректального рака [18-20] (табл. 1). MS-HRM проводили с использованием ПЦР-смеси, содержащей краситель EvaGreen, "Precision Melt Supermix" (Bio-Rad Laboratories, Inc., США). Температуры отжига были оптимизированы так, чтобы между кривыми плавления калибровочных образцов было чёткое разделение (табл. 1). Выбранную температуру отжига в дальнейшем использовали для постановки MS-HRM с образцами ДНК пациентов. Все реакции выполнялись в двух повторах. Уровень метилирования в образце определяли по графикам пиков плавления (melt peak) относительно калибровочных кривых (рис. 1). Каждый образец имел минимальное и максимальное значения уровня метилирования для каждого гена. Для статистической обработки вычисляли среднее значение уровня метилирования.

\section{Калибровочные кривые для MS-HRM}

Для получения калибровочных кривых плавления использовали подвергнутые бисульфитной конверсии образцы полностью метилированной ДНК $\mathrm{CpG}$ Methylated Human genomic DNA ("Thermo Fisher Scientific", США) (К+) и ДНК из периферической крови здорового мужчины - неметилированная контрольная ДНК (K-). К+ и $\mathrm{K}$ - смешивались в процентном соотношении по массе $\mathrm{K}+/ \mathrm{K}-$ : 0/100, 5/95, 10/90, 20/80, 30/70, 50/50, 75/25 и 100/0, а уровень метилирования для каждого калибровочного образца был равен $0 ; 0,05 ; 0,1 ; 0,2 ; 0,3 ; 0,5 ; 0,75$ и 1 соответственно. 
Скородумова и др.

Таблица 1. Последовательности используемых в работе праймеров и их характеристики

\begin{tabular}{|c|c|c|c|c|}
\hline $\begin{array}{c}\text { Название } \\
\text { гена }\end{array}$ & Последовательности праймеров & $\begin{array}{c}\text { Координаты начала } \\
\text { ампликона (GRCh37) }\end{array}$ & $\begin{array}{c}\text { Координаты конца } \\
\text { ампликона (GRCh37) }\end{array}$ & $\mathrm{Ta},{ }^{\circ} \mathrm{C}$ \\
\hline \multirow{2}{*}{ GSTP1 } & 5'-GAGAAGTACGAGATGTGGGGAT-3' & \multirow{2}{*}{67351855} & \multirow{2}{*}{67351922} & \multirow{2}{*}{59,0} \\
\hline & 5'-TACACTCCТAACСCCTCCCC-3' & & & \\
\hline \multirow{2}{*}{ APC } & 5'-CGGGGTTTTGTGTTTTATTG-3' & \multirow{2}{*}{112073406} & \multirow{2}{*}{112073477} & \multirow{2}{*}{58,7} \\
\hline & 5'-TCCAACGAATTACACAACTAC-3' & & & \\
\hline \multirow{2}{*}{ RASSF1 } & 5'-GTCGGGGTTTGTTTTGTGGTT-3' & \multirow{2}{*}{50378315} & \multirow{2}{*}{50378198} & \multirow{2}{*}{54,5} \\
\hline & 5'-CAACTCCCACAACTCAATAAACT-3' & & & \\
\hline
\end{tabular}
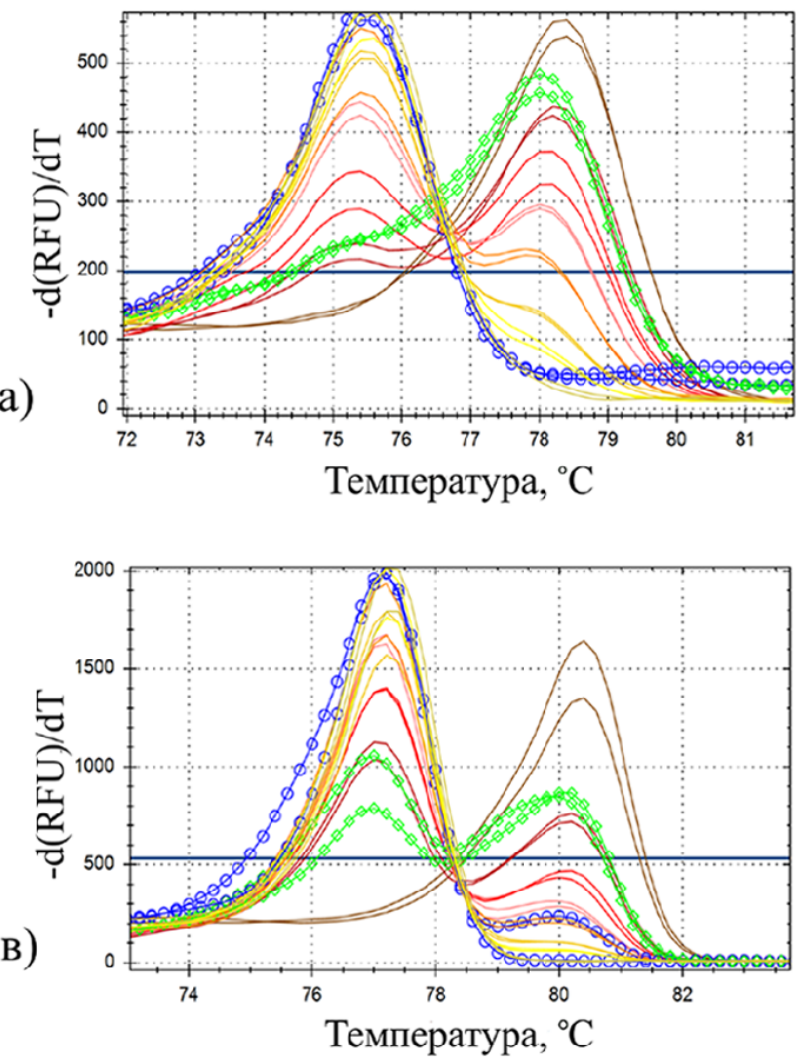

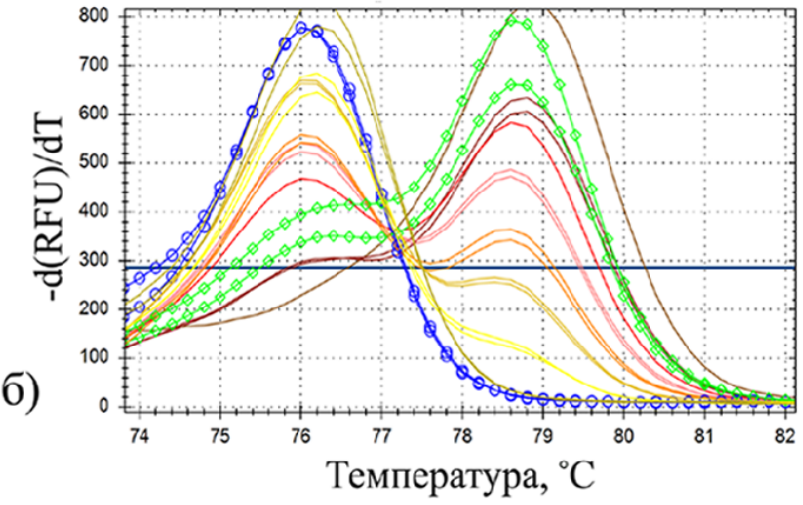

Калибровочные кривые: $\mathrm{K}+/ \mathrm{K}-$

Образцы: - - "норма" $-0 / 100$

$-5 / 95$

$-10 / 90$

$--20 / 80$

$--30 / 70$

$--50 / 50$

$--25 / 75$

$--100 / 0$

Рисунок 1. Пример результатов анализа метилирования исследуемых генов с помощью MS-HRM: a) GSTP1, б) APC, в) RASSF1.

\section{Статистическая обработка данных} MS-HRM u HM450

Для проверки гипотезы о нормальном распределении значений выборки по критерию Д'Агостино-Пирсона, для расчёта регрессии по методу Пассинга-Баблока, для построения графиков Блэнда-Алтмана использовали программу "MedCalc", версия 16.4.3. (“MedCalc Software bvba”, Бельгия).

\section{Анализ метилирования на чипе НM450}

Исследование количественного статуса метилирования было произведено с использованием набора Infinium HumanMethylation450 BeadChip array ("Illumina, Inc.", США) согласно инструкции производителя. Парные образцы были представлены в пределах одного чипа. Контроль качества и препроцессинг данных, полученных при использовании чипа НМ450, проводили для двух чипов одновременно при помощи программного обеспечения
RnBeads v0.99 [21]. Фильтрацию корректно идентифицированных $\mathrm{CpG}$ сайтов производили с использованием алгоритма Greedycut, со следующими показателями: $p>0,05$, покрытие $<95 \%$ [21]. В качестве меры метилирования использовалась количественная величина $\beta$, вычисляемая по формуле:

$$
\beta=\frac{\text { Imet }}{\text { Imet }+ \text { Inonmet }}
$$

где Imet - интенсивность сигнала от зонда, идентифицирующего определенный метилированный CpGcaйт, a Inonmet - интенсивность от зонда, идентифицирующего этот же $\mathrm{CpG}$ сайт в случае его неметилированного статуса. Таким образом, диапазон значений $\beta$ меняется от 0 до 1 , где $\beta=0$ говорит об отсутствии метилирования в исследуемом сайте, а $\beta=1-$ о его полном метилировании. Фоновую коррекцию и нормализацию данных производили с использованием алгоритмов NOOB и BMIQ [22, 23]. 


\section{РЕЗУЛЬТАТЫ И ОБСУЖДЕНИЕ}

Различия между образиами опухолевой ткани и “нормы” при РПЖ по уровню метилирования ДНК, определяемого методом MS-HRM

Потенциал диагностического анализа определяется в частности его способностью дифференцировать заявленные группы образцов. В данном случае было необходимо оценить, различаются ли образцы опухолевой ткани и “нормы” при РПЖ по уровню метилирования генов GSTP1, APC и RASSF1, определенному с помощью MS-HRM. Средние значения уровней метилирования для каждого образца приведены в таблице 2. Для сравнения двух групп в первую очередь была проверена нормальность распределения значений уровней метилирования ДНК с помощью теста Д'Агостино-Пирсона в каждой из выборок. Нормальность была подтверждена для всех выборок, кроме значений метилирования гена GSTP1 в группе образцов “нормы”. Так как при выбранной калибровке невозможно оценить различия уровня метилирования ДНК в диапазоне от 0 до 0,05 , то отсутствием нормального распределения для данной выборки пренебрегли. Различие группы образцов опухолевой ткани и “нормы" по значениям метилирования для каждого из генов было подтверждено с помощью критерия Стьюдента $(\alpha=0,001)$ и метода доверительных интервалов $(\alpha=0,05)$. Для всех групп сравнения значения критерия Стьюдента были выше критического при заданном уровне значимости $\left(\mathrm{t}_{\mathrm{a}}=2,921\right)$, и $95 \%$ доверительные интервалы не пересекались (табл. 3).

Таким образом, анализ метилирования генов GSTP1, APC и RASSF1 методом MS-HRM с помощью подобранных пар праймеров и условий реакции позволяет достоверно различить образцы опухолевой ткани РПЖ и “нормы”.

Анализ метилирования генов GSTP1, APC и RASSF1 в образиах опухолевой ткани и “нормьл" при РПЖ с помощьью чипа НM450

Для образцов, полученных от больных РПЖ, было также исследовано метилирование ДНК с помощью гибридизации на чипах. Мы проверили, различаются ли образцы опухолевой ткани и "нормы" при РПЖ по уровням метилирования генов GSTP1, APC и RASSF1 по данным чипа HM450. Для статистических вычислений были использованы значения $\beta$ зондов, координаты которых лежат внутри координат начала и конца ампликонов MS-HRM (табл. 2). Для гена GSTP1 были взяты значения наиболее близкого зонда c координатой 67351786.В границы координат ампликона гена АРС попадают несколько зондов микроматричного чипа, поэтому в статистических расчетах использовали среднее значений $\beta$. C помощью теста Д'Агостино-Пирсона была подтверждена нормальность распределения значений уровней метилирования для всех выборок. Группы образцов опухолевой ткани и “нормы" достоверно различались по значениям метилирования для каждого из генов по критерию Стьюдента $(\alpha=0,001)$ и доверительным интервалам $(\alpha=0,05)$ (табл. 3$)$.

Таким образом, различия по уровню метилирования ДНК между образцами опухолевой ткани и “нормы” при РПЖ, выявленные методом MS-HRM, подтверждаются методом чип-гибридизации.

Таблица 2. Уровни метилирования генов GSTP1, APC и RASSF1, в образцах опухолевой ткани и “нормы” при РПЖ, определённые с помощью MS-HRM или гибридизации на чипах HM450

\begin{tabular}{|c|c|c|c|c|c|c|}
\hline \multicolumn{7}{|c|}{ Опухолевая ткань } \\
\hline \multirow{2}{*}{ Название образца } & \multicolumn{2}{|c|}{ GSTP1 } & \multicolumn{2}{|c|}{ APC } & \multicolumn{2}{|c|}{ RASSF1 } \\
\hline & MS-HRM & HM450 & MS-HRM & HM450 & MS-HRM & HM450 \\
\hline PC1019 & 0,3 & 0,6546 & 0,4 & 0,7311 & 0,7 & 0,5795 \\
\hline PC1035 & 0,3 & 0,7646 & 0,325 & 0,5467 & 0,575 & 0,6626 \\
\hline PC1037 & 0,5 & 0,6867 & 0,625 & 0,6834 & 0,725 & 0,4683 \\
\hline PC1057 & 0,15 & 0,7569 & 0,35 & 0,8222 & 0,55 & 0,4854 \\
\hline PC1063 & 0,825 & 0,8289 & 0,875 & 0,8529 & 0,825 & 0,7032 \\
\hline PC1067 & 0,15 & 0,564 & 0,575 & 0,7208 & 0,675 & 0,5611 \\
\hline PC1071 & 0,25 & 0,6228 & 0,34 & 0,5703 & 0,525 & 0,6125 \\
\hline PC1075 & 0,4 & 0,6583 & 0,35 & 0,6493 & 0,6 & 0,5332 \\
\hline \multicolumn{7}{|c|}{ «Норма» } \\
\hline \multirow{2}{*}{ Название образца } & \multicolumn{2}{|c|}{ GSTP1 } & \multicolumn{2}{|c|}{$\mathrm{APC}$} & \multicolumn{2}{|c|}{ RASSF1 } \\
\hline & MS-HRM & HM450 & MS-HRM & HM450 & MS-HRM & HM450 \\
\hline PC1020 & 0,05 & 0,0576 & 0 & 0,0362 & 0,025 & 0,077 \\
\hline PC1036 & 0,05 & 0,0675 & 0 & 0,0666 & 0,025 & 0,2295 \\
\hline PC1038 & 0,05 & 0,1664 & 0,05 & 0,1219 & 0,075 & 0,1358 \\
\hline PC1058 & 0,05 & 0,081 & 0 & 0,0678 & 0,15 & 0,1287 \\
\hline PC1064 & 0,05 & 0,1234 & 0 & 0,0399 & 0,125 & 0,1218 \\
\hline PC1068 & 0,05 & 0,0429 & 0 & 0,0423 & 0,075 & 0,046 \\
\hline PC1072 & 0,05 & 0,3691 & 0,05 & 0,0778 & 0,15 & 0,3254 \\
\hline PC1076 & 0,05 & 0,2476 & 0 & 0,0598 & 0,075 & 0,0907 \\
\hline
\end{tabular}


Скородумова и др.

Таблица 3. Статистические показатели и результаты регрессионного анализа по методу Пассинга-Баблока, характеризующие выборку образцов опухолевой ткани (“О”) и “нормы” (“Н”) при РПЖ

\begin{tabular}{|c|c|c|c|c|}
\hline & \multicolumn{4}{|c|}{ GSTP1 } \\
\hline Вид анализа & \multicolumn{2}{|c|}{ HM450 } & \multicolumn{2}{|c|}{ MS-HRM } \\
\hline Вид образца & $\mathrm{O}$ & $\mathrm{H}$ & $\mathrm{O}$ & $\mathrm{H}$ \\
\hline Среднее & 0,69211 & 0,14445 & 0,35938 & 0,05 \\
\hline $\begin{array}{l}\text { Стандартное } \\
\text { отклонение }\end{array}$ & 0,08616 & 0,11329 & 0,22198 & 0 \\
\hline $\begin{array}{c}\text { Стандартная ошибка } \\
\text { среднего }\end{array}$ & 0,03046 & 0,04005 & 0,07848 & 0 \\
\hline 95CI ${ }^{1}$ нижняя граница & 0,62524 & 0,05653 & 0,18711 & 0,05 \\
\hline 95С ${ }^{1}$ верхняя граница & 0,75897 & 0,23236 & 0,53164 & 0,05 \\
\hline $\mathrm{P}^{2}$ & 0,9241 & 0,1516 & 0,0517 & $\mathrm{SD}=0$ \\
\hline $\mathrm{t}^{3}$ & \multicolumn{2}{|c|}{10,8832} & \multicolumn{2}{|c|}{3,942} \\
\hline $\begin{array}{c}\text { Регрессионный } \\
\text { анали }^{4}\end{array}$ & \multicolumn{4}{|c|}{$y=0,0118198+0,792570 x$} \\
\hline & \multicolumn{4}{|c|}{ APC } \\
\hline Вид анализа & \multicolumn{2}{|c|}{ HM450 } & \multicolumn{2}{|c|}{ MS-HRM } \\
\hline Вид образца & $\mathrm{O}$ & $\mathrm{H}$ & $\mathrm{O}$ & $\mathrm{H}$ \\
\hline Среднее & 0,6971 & 0,06402 & 0,48 & 0,0125 \\
\hline $\begin{array}{l}\text { Стандартное } \\
\text { отклонение }\end{array}$ & 0,10878 & 0,02779 & 0,19636 & 0,02315 \\
\hline $\begin{array}{c}\text { Стандартная ошибка } \\
\text { среднего }\end{array}$ & 0,03846 & 0,00983 & 0,06942 & 0,00818 \\
\hline 95С ${ }^{1}$ нижняя граница & 0,61268 & 0,04245 & 0,32762 & $-0,00546$ \\
\hline 95С ${ }^{1}$ верхняя граница & 0,78151 & 0,08558 & 0,63238 & 0,03046 \\
\hline $\mathrm{P}^{2}$ & 0,839 & 0,0743 & 0,1281 & 0,1594 \\
\hline $\mathrm{t}^{3}$ & 15,9 & 493 & 6,68 & 877 \\
\hline \multirow[t]{2}{*}{$\begin{array}{l}\text { Регрессионный } \\
\text { анализ }^{4}\end{array}$} & \multicolumn{4}{|c|}{$y=0,336121+0,576645 x$} \\
\hline & \multicolumn{4}{|c|}{ RASSF1 } \\
\hline Вид анализа & \multicolumn{2}{|c|}{ HM450 } & \multicolumn{2}{|c|}{ MS-HRM } \\
\hline Вид образца & $\mathrm{O}$ & $\mathrm{H}$ & $\mathrm{O}$ & $\mathrm{H}$ \\
\hline Среднее & 0,57573 & 0,14435 & 0,64688 & 0,0875 \\
\hline $\begin{array}{c}\text { Стандартное } \\
\text { отклонение }\end{array}$ & 0,08183 & 0,09098 & 0,10215 & 0,05 \\
\hline $\begin{array}{c}\text { Стандартная ошибка } \\
\text { среднего }\end{array}$ & 0,02893 & 0,03217 & 0,03612 & 0,01768 \\
\hline 95CI ${ }^{1}$ нижняя граница & 0,51223 & 0,07375 & 0,5676 & 0,0487 \\
\hline 95 $\mathrm{CI}^{1}$ верхняя граница & 0,63924 & 0,21495 & 0,72615 & 0,1263 \\
\hline $\mathrm{P}^{2}$ & 0,8236 & 0,1378 & 0,7386 & 0,5631 \\
\hline $\mathrm{t}^{3}$ & \multicolumn{2}{|c|}{9,9711} & \multicolumn{2}{|c|}{13,9111} \\
\hline $\begin{array}{l}\text { Регрессионный } \\
\text { анализ }^{4}\end{array}$ & \multicolumn{4}{|c|}{$y=0,299724+0,739748 x$} \\
\hline
\end{tabular}

Примечание: 1 - 95СI - 95\% доверительный интервал при уровне значимости $\alpha=0,05 ; 2$ - значение критерия Д'Агостино-Пирсона, SD=0 - распределение не нормально; 3 - значение критерия Стьюдента при уровне значимости $\alpha=0,001 ; 4$ - уравнение регрессионного анализа по методу Пассинга-Баблока при сравнении разностей уровней метилирования в опухолевой ткани и “норме", определённых методом HM450 и MS-HRM.
Согласованность разностей уровней метилирования генов GSTP1, APC и RASSF1, определенных в каждой паре образиов опухолевая ткань/“норма”, методами гибридизации на чипах и $M S-H R M$

С диагностической точки зрения самым важным при оценке результатов метилирования ДНК является выраженное различие (разность) между уровнями метилирования не суммарно в группе образцов опухолевой ткани или "нормы", а в каждой паре образцов опухолевая ткань/“норма”. Поэтому была оценена согласованность разностей уровней метилирования (РУМ), полученных методом гибридизации на чипах или методом MS-HRM. Для каждого пациента РУМ вычисляли как разность между уровнем метилирования ДНК в образце опухолевой ткани и в соответствующем ему образце “нормы”. Для оценки согласованности РУМ были использованы регрессионный анализ Пассинга-Баблока и графики Блэнда-Алтмана $[24,25]$.

Анализ по методу Пассинга-Баблока, результаты которого приведены в таблице 3, показал, что для RASSF1 РУМ между двумя методами хорошо согласуются: уравнение регрессии свидетельствует о небольшом вкладе систематической ошибки, а показатель корреляции близок к 1. По уравнению регрессии для АРС можно заключить, что существует систематическая ошибка, и показатель корреляции имеет среднее значение. Для GSTP1 также выявлен вклад систематической ошибки, но показатель корреляции близок к 1.

Графики, полученные в результате анализа согласования РУМ по методу Блэнда-Алтмана, приведены на рисунке 2. Для генов GSTP1 и APC подтверждаются систематические ошибки, обнаруженные в результатах анализа по ПассингуБаблоку: значения РУМ, определяемые с помощью MS-HRM, были в среднем на 0,24 и 0,17 меньше, чем значения РУМ по данным чип-гибридизации соответственно. Для GSTP1 эта ошибка больше; это может быть объяснено тем, что результаты MS-HRM сравниваются с данными зонда, не входящего в координаты границ ампликона, а расположенного на расстоянии в 69 нуклеотидов от него, либо особенностями праймеров, которые могут приводить к предпочтительной амплификации неметилированной ДНК. Тем не менее, границы 95\% доверительного интервала для средних значений РУМ лежат очень близко к линиям равнозначности. По графику анализа РУМ гена RASSF1 наблюдается небольшая систематическая ошибка: завышение РУМ методом MS-HRM на 0,13. Точки распределены близко линии средней разности РУМ, и такой характер распределения (за исключением одного образца) сохраняется как при малых, так и больших значениях разности (рис. 2в).

Суммируя вышесказанное, можно заключить, что с поправкой на статистическое занижение или завышение PУM, данные MS-HRM удовлетворительно или хорошо согласуются с результатами НМ450. Неточная согласованность РУМ может быть объяснена тем, что результаты MS-HRM представляют собой интегральное значение 
a)

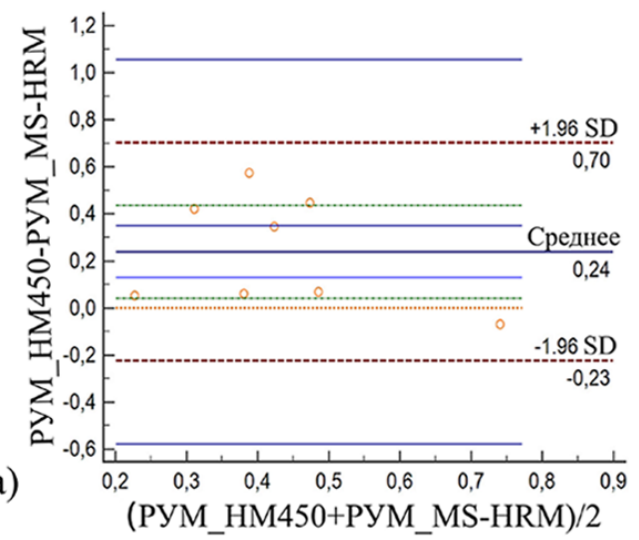

B)

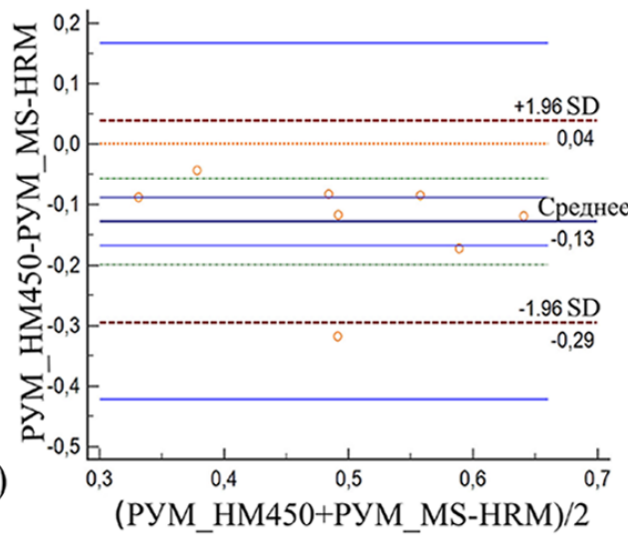

б)

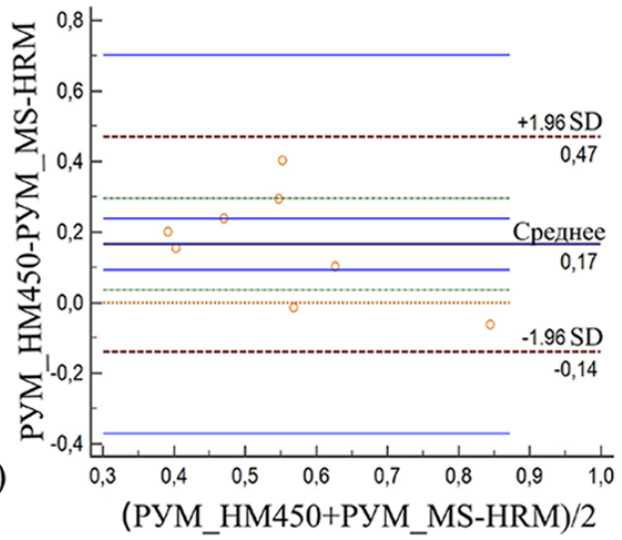

Условные обозначения:

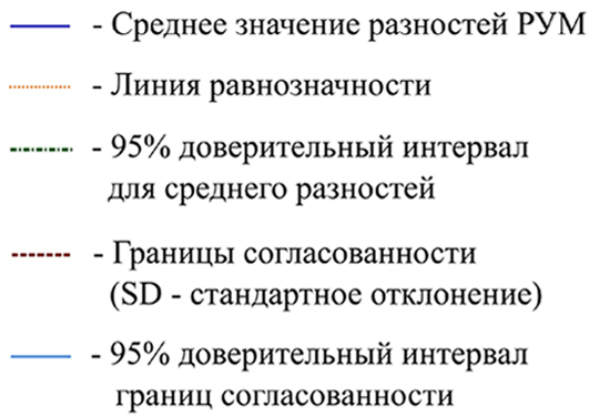

Рисунок 2. Анализ разности уровней метилирования ДНК исследуемых генов, определяемых методами гибридизации на чипах HM450 (PУM_HM450) и MS-HRM (PYM_MS-HRM): a) GSTP1, б) APC, в) RASSF1.

метилированности всех $\mathrm{CpG}$ сайтов, попадающих в ампликон, а значения уровня метилирования чипа представляют собой дискретный сигнал, чаще всего с одного зонда. Кроме того, для MS-HRM в качестве калибровочных образцов используются смеси полностью метилированной ДНК с полностью неметилированной, тогда как в амплифицируемом участке ДНК образца, скорее всего, часть CpG сайтов метилирована, а часть - нет. Возможно, всё это влияет на выраженность пика кривой плавления.

Согласование РУМ между данными MS-HRM и гибридизации на чипе указывает на возможность использования данных о дифференциально метилированных сайтах с чипа для поиска потенциальных маркеров РПЖ. Не исключено, что расположение ампликона на некотором расстоянии от зонда снижает степень согласованности PУМ, как это было отмечено для гена GSTP1. В связи с этим, была выдвинута гипотеза о том, что для адекватного воспроизведения результатов чип-гибридизации методом MS-HRM необходимо позиционировать праймеры в дифференциально метилированных регионах.

\section{ЗАКЛЮЧЕНИЕ И ВЫВОДЫ}

В данной работе было впервые исследовано метилирование генов GSTP1, APC и RASSF1 с помощью MS-HRM в образцах опухолевой ткани и “нормы” при РПЖ. Метод MS-HRM позволяет достоверно различить группы образцов опухолевой ткани и “нормы” по уровню метилирования ДНК каждого из генов. Результаты анализа метилирования генов GSTP1, APC и RASSF1 с помощью MS-HRM были сопоставлены с результатами гибридизации на чипах. Данные гибридизации на чипах подтвердили разделение групп образцов опухолевой ткани и “нормы” при РПЖ, выявленное с помощью MS-HRM. Кроме того, с поправкой на небольшую систематическую ошибку, значения РУМ для каждого образца, определённые MS-HRM и чип-гибридизацией, согласовались друг с другом. Таким образом, есть основания полагать, что оценка уровня метилирования генов GSTP1, APC и RASSF1 с помощью MS-HRM подходит для разработки лабораторного теста, уточняющего диагностику РПЖ.

Работа выполнена при финансовой поддержке Министерства образования и науки РФ, соглашение о предоставлении субсидии № 14.607.21.0068 от 23 сентября 2014 г., уникальный идентификатор ПНИ RMЕFI60714X0068.

\section{ЛИТЕРАТУРА}

1. Ferlay J., Soerjomataram I., Dikshit R., Eser S., Mathers C., Rebelo M., Parkin D.M., Forman D., Bray F. (2015) Int. J. Cancer, 136(5), E359-E386.

2. Каприн А.Д., Старинский В.В., Петрова Г.В. (2016) Состояние онкологической помощи населению России в 2015 году, ФГУ “МНИОИ им. П.А. Герцена Росмедтехнологий”, М., 236 с. 
3. Humphrey P.A., Moch H., Cubilla A.L., Ulbright T.M., Reuter V.E. (2016) Eur. Urol., 70(1), 106-119.

4. Van Neste L., Partin A. W., Stewart G.D., Epstein J.I., Harrison D.J., Van Criekinge W. (2016) Prostate, 76(12), 1078-1087.

5. Hanahan D., Weinberg R.A. (2011) Cell, 144(5), 646-674.

6. Berdasco M., Esteller M. (2010) Dev. Cell, 19(5), 698-711.

7. Rybicki B.A., Rundle A., Kryvenko O.N., Mitrache N., Do K.C., Jankowski M., Chitale D.A., Trudeau S., Belinsky S.A., Tang D. (2016) Int. J. Cancer, 138(12), 2884-2893.

8. Yang B., Bhusari S., Kueck J., Weeratunga P., Wagner J., Leverson G., Huang W., Jarrard D.F. (2013) Neoplasia, 15(4), 399-408.

9. Kobayashi Y., Absher D.M., Gulzar Z.G., Young S.R., McKenney J.K., Peehl D.M., Brooks J.D., Myers R.M., Sherlock G. (2011) Genome Res., 21(7), 1017-1027.

10. Mahapatra S., Klee E.W., Young C.Y., Sun Z., Jimenez R.E., Klee G.G., Tindall D.J., Donkena K.V. (2012) Clin. Cancer Res., 18(10), 2882-2895.

11. Kim J.W., Kim S.T., Turner A.R., Young T., Smith S., Liu W., Lindberg J., Egevad L., Gronberg H., Isaacs W.B., Xu J. (2012) PloS One, 7(10), e48455.

12. Haldrup C., Mundbjerg K., Vestergaard E.M., Lamy P., Wild P., Schulz W.A., Arsov C., Visakorpi T., Borre M., Høyer S., Ørntoft T.F., Surensen K.D. (2013) J. Clin. Oncol., 31(26), 3250-3258.

13. Geybels M.S., Zhao S., Wong C.J., Bibikova M., Klotzle B., Wu M., Ostrander E.A., Fan J.B., Feng Z., Stanford J.L. (2015) Prostate, 75(16), 1941-1950.

14. Hessels D., Schalken J.A. (2013) Asian J. Androl., 15(3), 333-339.
15. Henrique R., Ribeiro F.R., Fonseca D., Hoque M.O., Carvalho A.L., Costa V.L., Pinto M., Oliveira J., Teixeira M.R., Sidransky D., Jerónimo C. (2007) Clin. Cancer Res., 13(20), 6122-6129.

16. Litovkin K., Van Eynde A., Joniau S., Lerut E., Laenen A., Gevaert T., Spahn M., Kneitz B., Gramme P., Helleputte T., Isebaert S., Haustermans K., Helleputte T. (2015) PloS One, 10(6), e0130651.

17. Бабалян К.А., Султанов Р., Генерозов Е.В., Захаржевская Н.Б., Шарова Е.И., Пешков М.Н., Васильев А.О., Говоров А.В., Пушкарь Д.Ю., Прилепская Е.А., Даниленко С.А., Бабикова Е.А., Ларин А.К., Говорун В.М. (2016) Вопросы онкологии, №1, 122-132 .

18. Wojdacz T.K., Dobrovic A., Hansen L.L. (2008) Nat. Protoc., 3(12), 1903-1908.

19. Spitzwieser M., Holzweber E., Pfeiler G., Hacker S., Cichna-Markl M. (2015) Breast Cancer Res., 17, 125.

20. Migheli F., Stoccoro A., Coppede F., Omar W.A.W., Failli A., Consolini R., Seccia M., Spisni R., Miccoli P., Mathers J.C., Migliore L. (2013) PLoS One, 8(1), e52501.

21. Assenov Y., Müller F., Lutsik P., Walter J., Lengauer T., Bock C. (2014) Nat. Methods, 11(11), 1138-1140.

22. Teschendorff A.E., Marabita F., Lechner M., Bartlett T., Tegner J., Gomez-Cabrero D., Beck S. (2013) Bioinformatics, 29(2), 189-196.

23. Triche T.J., Weisenberger D.J., Van Den Berg D., Laird P.W., Siegmund K.D. (2013) Nucleic Acids Res., 41(7), e90.

24. Bilic-Zulle L. (2011) Biochem. Med. (Zagreb), 21(1), 49-52.

25. Giavarina D. (2015) Biochem. Med. (Zagreb), 25(2), 141-151.

Поступила: $\quad 30.09 .2016$. Принята к печати: 29. 11. 2016.

\title{
GSTP1, APC AND RASSF1 GENE METHYLATION IN PROSTATE CANCER SAMPLES: COMPARATIVE ANALYSIS OF MS-HRM METHOD AND INFINIUM HUMANMETHYLATION450 BEADCHIP ARRAY DIAGNOSTIC VALUE
}

\author{
L.O. Skorodumova', K.A. Babalyan', R. Sultanov', A.O. Vasiliev, A.V. Govorov , D.Y. Pushkar, \\ E.A. Prilepskaya ${ }^{3}$, S.A. Danilenko ${ }^{1}$, E.V. Generozov', A.K. Larin ${ }^{1}$, E.S. Kostryukova ${ }^{1,2}$, E.I. Sharova ${ }^{1}$
}

${ }^{1}$ Federal Research and Clinical Center of Physical-Chemical Medicine,

1a Malaya Pirogovskaya str., Moscow, 119435 Russia; tel.: +7(499)2464501; fax: +7(499)2464409; e-mail: 1.skorodumova@rcpcm.org

${ }^{2}$ Moscow Institute of Physics and Technology, Dolgoprudniy, Moscow Region, Russia ${ }^{3}$ Moscow State Medical Stomatological University, Moscow, Russia

There is a clear need in molecular markers for prostate cancer (PC) risk stratification. Alteration of DNA methylation is one of processes that occur during PC progression. Methylation-sensitive PCR with high resolution melting curve analysis (MS-HRM) can be used for gene methylation analysis in routine laboratory practice. This method requires very small amounts of DNA for analysis. Numerous results have been accumulated on DNA methylation in PC samples analyzed by the Infinium HumanMethylation450 BeadChip (HM450). However, the consistency of MS-HRM results with chip hybridization results has not been examined yet. The aim of this study was to assess the consistency of results of GSTP1, APC and RASSF1 gene methylation analysis in PC biopsy samples obtained by MS-HRM and chip hybridization. The methylation levels of each gene determined by MS-HRM were statistically different in the group of PC tissue samples and the samples without signs of tumor growth. Chip hybridization data analysis confirmed the results obtained with the MS-HRM. Differences in methylation levels between tumor tissue and histologically intact tissue of each sample determined by MS-HRM and chip hybridization, were consistent with each other. Thus, we showed that the assessment of GSTP1, APC and RASSF1 gene methylation analysis using MS-HRM is suitable for the design of laboratory assays that will differentiate the PC tissue from the tissue without signs of tumor growth.

Key words: prostate cancer, DNA methylation, HRM, GSTP1, APC, RASSF1 